\title{
Role of Preoperative Fascia Iliaca Compartment Block On The Side Of Femur Surgery
}

\author{
Radhashyam Paria ${ }^{1}$, Smarajit Surroy ${ }^{1}$, Mousumi Majumder ${ }^{1}$, Baishakhi Paria ${ }^{2}$, \\ Soma Sengupta ${ }^{3}$, GoutamDas, ${ }^{4}$ Anshuman Paria ${ }^{5}$ \\ 1(Dept of Anesthesiology, Howrah Orthopedic Hospital, Eastern Railways, WB) \\ 2(Department of Community Medicine, National Medical College) \\ 3(dept of Anesthesiology, Columbia Asia Hospital, Salt Lake, WB) \\ 4(Department of Pediatric Medicine, North Bengal Medical College, West Bengal) \\ 5(dept of Neonatology, SSKM hospital, WB)
}

\begin{abstract}
:
Background and Aims: Different kinds of adjutants are used with local anesthetics during intrathecal procedure for prolongation of action. However, they are not completely free from side effects. Fascia Iliaca Compartment Block (FICB) is useful for the same purpose.

Method: After administering 20ml 0.5\% injection Bupivacaine in FIC (Fascia Iliaca Compartment) on the fractured side before anesthesia as pre-operative medication, 50 elderly patients got relief of pain.

Results: 50 patients of mean age and weight $68.38 \pm 8.08$ years and $55.04 \pm 3.02 \mathrm{Kg}$ respectively showed postoperative analgesia of average 15.82 \pm 2.34 hours with mean systolic blood pressure $112.32 \pm 7.62 \mathrm{~mm}$ of $\mathrm{Hg}$. Patients felt comforts with FICB.

Conclusion: Preoperative, intra-operative and postoperative analgesia provided by FICB is of high quality to relieve pain on movement before surgery, to act as an adjuvant with spinal anesthesia during surgery and prolonged analgesia after surgery without adverse reaction.
\end{abstract}

\section{Introduction}

Excruciating pain at fracture site and its exaggeration on movement during transport and intrathecal procedure are the main culprits for increasing stress, strain and mental agony of the patients. Profound analgesia is the essential component of anesthesia to control surgical stress response both during and after surgery. In relation to pre-operative medication, the usual use of systemic opioid frequently relieves pain with a lot of side effects particularly in the older group of patients. However, in search of good analgesic without side effects, we hypothesized that fascia iliaca compartment block (FICB) may be perfectly eligible for the above purpose in case of femur fracture. Based on such assumption, we designed our study to administer (FICB) on fracture side of femur pre-operatively to evaluate its efficacy and effectiveness as preoperative, intraoperative and post-operative analgesic.

\section{Methods}

With written informed consent for procedure and study, we recruited 50 patients, aged between 60 to 90 years, with ASA physical status class II and III, undergoing femur surgery in the period of March 2013 to November 2013. At the time of preoperative assessment to eliminate the cases, contraindicated for spinal anesthesia, we examined the area surrounding the inguinal crease on the fracture side to exclude the local infection for further procedure like FICB.

In the operation room, after establishment of intravenous infusion and identification of anatomical landmarks like anterior superior iliac spine and pubic tubercle on the fractured side in the supine position of the patient we adopted aseptic preparation of the part. We located a point of $1 \mathrm{~cm}$ below from the junction of lateral one-third and medial two-third of the inguinal ligament and felt the pulsation of femoral artery. We inserted an intramuscular needle of $21 \mathrm{G}$ perpendicularly to the skin and advanced until we felt a loss of resistance as first click offered by fascia lata. With further progress of the needle, we experienced another loss of resistance as a second click offered by fascia iliaca and injected $20 \mathrm{ml}$ of $0.5 \%$ injection Bupivacaine. However, at the time of administration of the drug, we adopted precaution to avoid the intravascular injection by guarding the femoral artery and displacing medially by finger of the hand. With subsiding pain at the fractured site after administration of this block, we completed intrathecal procedure as usual in sitting position of the patient but without pain. 
Prior to the anesthetic procedure, we monitored every patient for non-invasive blood pressure, electrocardiogram, heart rate, and oxygen concentration and repeated at every 5 minutes. Intra-operative hemodynamic status and post-operative analgesic period were the main target to note for statistical analysis.

\section{Results:}

We administered FICB to 50 patients of the mean age and weight $68.38 \pm 8.08$ years and $55.04 \pm 3.02$ $\mathrm{Kg}$ for surgery femur. Their mean height was $157.96 \pm 4.63 \mathrm{~cm}$. Hemodynamic status including mean systolic blood pressure, heart rate and oxygen saturation was $112.32 \pm 7.62 \mathrm{~mm}$ of $\mathrm{Hg}, 89.92 \pm 7.62$ beats/minute and $98.94 \pm 0.89 \%$ respectively. Postoperative analgesia lasted for $15.82 \pm 2.34$ hours.

\section{Discussion}

Fracture pain is severe in nature, exaggerated on movement causing tremendous mental agony. For the relief of such excruciating pain at the fracture site, traditionally, we use systemic opioid although it is not free from side effects particularly in elderly patients. Other options to relieve such unbearable pain are less effective to comfort the patients. Analgesic effect of FICB is sufficient and superior at all time.

FICB is a method of peripheral nerve block for femoral, lateral cutaneous and obturator nerves and is responsible to offer analgesia for the area of the femur, hip and knee joint. It is a simple procedure, technically easy to perform and highly effective to provide analgesia before surgery as pre-operative medication for painless transport and intrathecal procedure. Its analgesic effect as an adjutant to spinal anesthesia in intra-operative period is more accountable and preferable than systemic opioid and even combined effect with epidural. Its analgesic effect to control surgical stress response during and after operative procedure in relation to the femur, hip and knee joint magnificently claims superiority in managing the deficits presented by spinal anesthesia.

The consideration of the anatomical configuration of the fascia iliaca (FI) covering the femoral nerve and its branches reveals that FI compartment is a potential space between FI and iliacus muscle itself. FI is located anterior to iliacus muscle within pelvis. The femoral nerve and lateral cutaneous nerve lie under this fascia whereas obturator nerve lies deep to iliacus muscle. The local anesthetic solution, deposited in this compartment beneath the FI should spread between this fascia and muscle. Femoral and lateral cutaneous nerves are involved quickly depending on volume of local anesthetic. The obturator nerve is not usually involved by FICB although this is not so important in pain relief of hip and knee. All the above mentioned nerves belong to lumbar plexus.

FICB provides analgesia of high quality ${ }^{1,2,3}$. The analgesic property of FICB is superior to systemic opioid, ${ }^{4,5}$ but inferior to epidural analgesia with fewer complications. ${ }^{6}$ Overall it is a very low risk procedure to block the femoral, obturator and lateral cutaneous nerves. The risk of intravascular injection, toxicity of local anesthetic and mechanical nerve damage is extremely low. The chance of infection is rare with good aseptic preparation of the site. Large volume of local anesthetic needed for FICB ensures good spread and reduces the risk of failure; however, the chance of drug toxicity is always present. So closed observation with monitoring at least for the first 15 minutes is essential and mandatory.

Most of the patients did not require systemic opioid at least for 15 hours in the postoperative period and when they required, they needed minimum dose. This duration of post operative analgesia is similar to Mulroy et al. ${ }^{7}$ The preoperative administration of FICB not only prolonged the postoperative analgesia, but also reduced further dose of systemic analgesic. The hemodynamic stability in perioperative period increases its further importance in clinical practice.

\section{Conclusion}

The analgesic property of FICB in case of femur surgeries is useful as pre-operative medication, adjutant to spinal anesthesia and post-operative analgesic to control surgical stress response particularly in elderly patients.

\section{Reference}

[1]. Vloka J, Hadzic A, Drobnik L, Ernest A, Reiss W, Thys D. Anatomical landmarks for femoral nerve block: a comparison of four needle insertion sites. Anesthesia \& Analgesia. 1999;89(6):1467.

[2]. Dahl J, Christiansen C, Daugaard J, Schultz P, Carlsson P. Continuous blockade of the lumbar plexus after knee surgerypostoperative analgesia and bupivacaine plasma concentrations. Anaesthesia. 1988;43(12):1015--1018

[3]. Edwards N, Wright E. Continuous low-dose 3-in-1 nerve blockade for postoperative pain relief after total knee replacement. Anesthesia \& Analgesia. 1992;75(2):265--267.

[4]. Matheny J, Hanks G, Rung G, Blanda J, Kalenak A. A comparison of patient-controlled analgesia and continuous lumbar plexus block after anterior cruciate ligament reconstruction. Arthroscopy: The Journal of Arthroscopic \& Related Surgery. 1993;9(1):87-90.

[5]. Serpell M, Millar F, Thomson M. Comparison of lumbar plexus block versus conventional opioid analgesia after total knee replacement. Anaesthesia. 1991;46(4):275--277. 
[6]. Schultz P, Christensen E, Anker-Moller E, Spangsberg N, Dahl J, Fauno P. Postoperative pain treatment after open knee surgery: continuous lumbar plexus block with bupivacaine versus epidural morphine. Regional Anesthesia and Pain Medicine. 1991;16(1):34--37.

[7]. Mulroy M, Larkin K, Batra M, Hodgson P, Owens B. Femoral nerve block with $0.25 \%$ or $0.5 \%$ bupivacaine improves postoperative analgesia following outpatient arthroscopic anterior cruciate ligament repair. Regional anesthesia and pain medicine. 2001;26(1):24-29 .

Table 1: Shows patient details along with hemodynamic status and Analgesic profile during the operation Demographic Profile

Age (Year)

$68.38 \pm 8.08$

Sex ( Male : Female)

Height $(\mathrm{cm})$

$157.96 \pm 4.67$

Weight $(\mathrm{Kg})$

$55.04 \pm 3.02$

Hemodynamic Profile:

$112.32 \pm 7.62$

Systolic Blood Pressure ( $\mathrm{mm} \mathrm{Hg}$ )

Oxygen Saturation (\%)

98.94 \pm 0.89

Heart Rate (beats / min).

$89.92 \pm 12.75$

Analgesic Profile

Postoperative analgesic(hours)

$15.82 \pm 2.34$ 